\title{
Sacralization and Herniated Nucleus Pulposus -An Association Study
}

Pradeep Kumar Sahoo, PP Mohanty* and Monalisa Pattnaik

Swami Vivekananda National Institute of Rehabilitation Training and Research, Cuttack - 754010, Odisha, India

\begin{abstract}
Introduction: Herniated nucleus pulposus (HNP) can be best diagnosed clinically by history followed by physical examinations suggested by Mckenzie and Cyriax as well as radiologically by MRI. Sacralization, which is mostly congenital in origin in later life, gives rise to altered biomechanics. It is thought to be a leading cause of low back pain and HNP. There is controversy in the literature whether sacralization is associated with HNP or not. So the present study intended to find out whether sacralization is associated with HNP.
\end{abstract}

Methodology: A total no of 150 subjects with LBP with or without radiation to lower limb were taken in the study. MRI report from each subject was studied. Subjects were diagnosed as HNP when they fulfill the clinical criteria as well as the MRI suggesting HNP. Sacralization was diagnosed by using lumbo-sacral A-P radiograph and various types of sacralization were noted. Pain was measured by using VAS, A-P diameter of spinal canal is noted from MRI and disability status was measured using ODI and WHODAS-2 (12 items).

Results: Result of the study showed that $71.42 \%$ sacralized subjects have HNP and sacralized subjects are 5.92 times risk for HNP, the relative risk factor for HNP in type $2 \mathrm{a}$ and $1 \mathrm{~b}$ is highest i.e., 9.44 each, next to it is type -4 i.e., 7.08 . The prevalence of LSTV was found to be $44 \%$ which includes sacralization $(42 \%)$ and lumbarisation $(2 \%)$. It has been found that incidence of sacralization in HNP group also found to be more i.e., $64.28 \%$. Besides this, it has also been found that sacralized subjects suffer from more disability and there is a weak correlation between SLR and disability. However, sacralization doesn't give rise to significant reduction of A-P diameter of spinal canal and more pain.

Conclusion: Sacralization is a risk factor for HNP and out of all sacralization type $-1 \mathrm{~B}$ and $2 \mathrm{~A}$ risk factor is highest. It has also been found that prevalence of sacralization in the low back pain population is more, sacralized individual suffered from more disability but not more pain or any changes in A-P diameter of spinal canal. SLR is weakly correlated with disability, A-P diameter of spinal canal and pain are not correlated.

Keywords: Low back pain; Herniated nucleus pulposus; Magnetic resonance imaging; Straight leg raising

Abbreviations: LBP-Low Back Pain; HNP-Herniated Nucleus Pulposus; MRI-Magnetic Resonance Imaging; ODI- Oswestry Low Back Pain Disability Questionnaire; LSTV- Lumbo Sacral Transitional Vertebra; SLR-Straight Leg Raising

\section{Introduction}

Low back pain (LBP) is an extremely common problem that most people experience at some point in their life [1]. The incidence of Herniated nucleus pulposus is 1-3\% [2]. Intervertebral disc being aneural is a predominant site for low back pain [3]. Herniated nucleus pulposus (HNP) can be best diagnosed clinically by history followed by physical examinations suggested by Mckenzie and Cyriax as well as radiologically by MRI. Bajpai et al. and Lunawat et al. [4] suggested HNP should be clinically correlated and clinician must put emphasis on history and physical examination. Enden and Palmer proved that even if abnormality present on MRI, not necessarily it is responsible for symptom.

According to Mckenzie [5] 1. Reduced lumbar lordosis, in 50\% cases associated with listing, 2. Repeated extension or repeated side gliding to affected side centralises the symptom suggestive of postreior derangement or HNP. According to Cyriax, [6] 1. Soft disc lesion (HNP) occours in $<60$ years of age 2 . Person assume a flexed/stoop posture suggestive of acute lumbago, deviate to opposite side if lesion is lateral to nerve root or deviate to affected side if lesion is medial to nerve root 3. Lumbar flexion,extension,side flexion to affected side is painful but side flexion to opposite side is relatively less painful. Spinal movements reproduce leg pain more than back pain 4 . SLR is limited. One of the common congenital anomaly commonly encountered at lumbo-sacral junction is lumbosacral transitional vertebra (LSTV), which include lumbarization and sacralization, 1st observed by Bertolotti. [7] The prevalence of LSTV in general population is found to be $4-35.9 \%$. Prevalence of lumbarization is varies in between $3.4-7.2 \%$ whereas sacralisation varies from $1.7-14 \%$ [8]. The sex distribution of lumbosacral anomaly shows greater incidence in men $(71.5 \%)$ than in women $(28.5 \%)$

Normally, the weight is transmitted by the fifth lumbar vertebra to sacum through unsacralised first sacral vertebra. In case of lumbar sacralization, weight transmission of trunk of human body takes place through LSTV. This creates a change in centre of gravity putting many anatomical structures under stress which may create multifold pathological problems such as irritation of sorrounding structures may be caused during rotation, forward/backward, lateral movements of trunk. Shifting of centre of gravity may force many structures to get overused causing addditional ossification creating other complications [9]. Whether sacralization predisposes to herniated nucleus pulposus is still unclear. Possible cause of disc herniation in unilateral sacralization are unilateral contact place put unusual stress on spine and results in torque movements cause herniation of disc one level above the sacralization [10].

Asymmetrical intervertebral joint articulation and movement in

*Corresponding author: Dr PP Mohanty Ph. D., Associate professor, Swam Vivekananda National Institute of Rehabilitation Training and Research, Cuttack - 754010, Odisha, India, Tel: +91-9437487139; Fax: +91-671-2805862; E-mail: ppmphysio@rediffmail.com

Received March 07, 2016; Accepted April 06, 2016; Published April 08, 2016

Citation: Sahoo PK, Mohanty PP, Pattnaik M (2016) Sacralization and Herniated Nucleus Pulposus -An Association Study. J Spine 5: 297. doi:10.4172/21657939.1000297

Copyright: ( 2016 Sahoo PK, et al. This is an open-access article distributed under the terms of the Creative Commons Attribution License, which permits unrestricted use, distribution, and reproduction in any medium, provided the original author and source are credited. 
lumbar spine put stress on disc, forcing fluid nucleus laterally lead to scoliosis [5]. Asymmetrical movement results in pressure exerted by nucleus in a part of annulus. This portion of annulus degenerate and tear prematurely lead to disc herniation [11]. Aihara et al. found that the iliolumbar ligament at the level immediately above is thinner and weaker whereas at the level of LSTV is broader and stronger. Because of this weak iliolumbar ligament the disc above the level of LSTV undergoes degeneration. Below to it stability is preserved due to bony union and protect disc from injury [12].

\section{Aim of the Study}

To find whether sacralization is a major risk factor for herniated nucleus pulposus.

\section{Methodology}

\section{Research design}

Association study

\section{Research setting}

The study was conducted in the Physiotherapy Department of SVNIRTAR, Olatpur, Cuttack.

\section{Sample size}

150 subjects

\section{Sampling method}

By convenient sampling the subjects were selected from low back pain population.

\section{Inclusion criteria}

Age between 15-75 years, Gender- both male and female, Low back pain with or without radiating pain to lower limb, Willing to participate in study.

\section{Exclusion criteria}

Spinal tumor, Spinal infection, Spinal trauma, Spondylolisthesis and spondylolysis, Post-operative subjects, Metabolic bone disease, Pregnancy, Other systemic diseases.

\section{Instrumentation}

Lumbo- sacral X-ray: A-P view, Magnetic resonance imaging, Universal Goniometre, McKenzie and Cyriax assessment protocol, Horizontal Visual analogue scale, Cotton, knee hammer for neurological examinations.

\section{Outcome measures}

Visual Analogue Scale (VAS), Straight leg raising (SLR): Hip flexion range angle in degree, WHODAS-2 (12 items), Oswestry disability index (ODI), Antero-posterior diameter of spinal canal in $\mathrm{cm}$.

\section{Procedure}

After fulfilling the inclusion and exclusion criteria subjects with low back pain with or without radiation reported to the Dept. of Physiotherapy, SVNIRTAR, Olatpur, Cuttack were taken in the study. A written informed consent was obtained from all the participants after their inclusion. Clinically McKenzie and Cyriax protocol was used and MRI of each subject was studied. HNP was diagnosed by both MRI and clinical correlation. Clinically, HNP was diagnosed using McKenzie and Cyriax protocol. The following clinical criteria should be present to diagnose HNP. Age $\leq 60$ years, reduced lumbar lordosis, listing may be present, Lumbar flexion, extension, side flexion to affected side limited and reproduce pain. Leg pain is more than back pain, repeated extension in prone centralize the pain, SLR is limited i.e., $\leq 60^{\circ}$. MRI report from each subject was studied. Subjects were diagnosed as HNP when they fulfill the clinical criteria as well as the MRI suggesting HNP. Sacralization was diagnosed by a radiologist of SCB Medical College and Hospital, Cuttack using Lumbo-sacral A-P radiograph and various types of sacralization was noted. Pain was measured by using VAS, A-P diameter of spinal canal was noted from MRI and disability status was measured using ODI and WHODAS-2 (12 items).

\section{Data Collection}

This was a prospective study. Data was collected in one day from each subject. Clinical examination was done using McKenzie and Cyriax protocol. MRI findings, presence of sacralization from X-ray, pain by VAS and disability by ODI, WHODAS-2 was recorded. All subjects were divided in to 4 groups. Group A- HNP + sacralisation, Group B- No HNP + sacralisation, Group C- HNP + No sacralisation and Group D - No HNP + No sacralisation.

\section{Data Analysis}

- Data analysis was done using SPSS version 16.

- Prevalence, proportion of sacralization, relative risk factor of sacralization was calculated.

- One way ANOVA was used to analyze pain, disability and A-P diameter of spinal canal between the 4 groups A, B, C, D.

- Tukey's post-hoc was used to analyse pain, disability, A-P diameter of spinal canal between the groups A, B, C, D with 0.05 level significance.

- Pearson correlation coefficient was used to find the correlation between A-P diameter of canal and pain, SLR and disability.

\section{Results}

A total no of 150 subjects with LBP were taken in the study as per inclusion and exclusion criteria. Out of the 150 subjects, 45 had both sacralisation and HNP, 18 had sacralizaion without HNP, 25 had HNP without sacralization and 59 had no sacralisation and HNP. 3 subjects presented with LBP had lumbarisation without HNP. They were divided in to 4 groups according to the presence or absence of HNP and sacralizaton, Group A- HNP + sacralisation, Group B- No HNP + sacralisation, Group C- HNP + No sacralisation, Group D- No HNP + No sacralisation (Graph 1).

\section{Proportion of sacralised subjects found to have HNP}

Proportion $=(45 / 45+18) \times 100 \%=71.42$

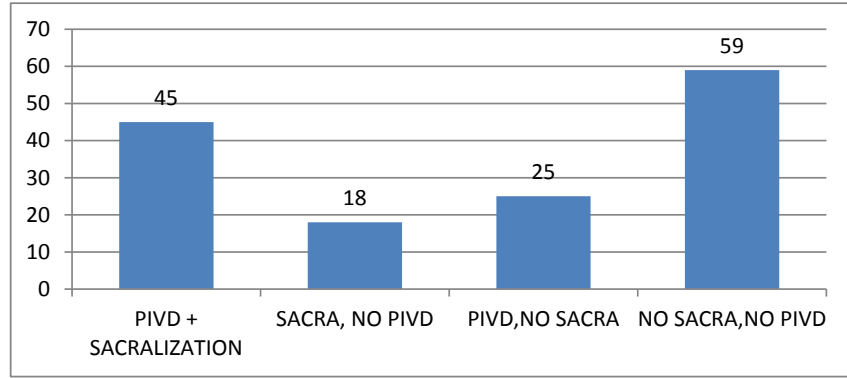

Graph 1: Distribution of low back pain subjects in 4 groups. 


\section{Prevalence of LSTV (Lumbosacral Transitional Vertebra)}

Prevalence of LSTV $=($ Existing cases/population examined at a given period of time $) \times 100 \%=(66 / 150) \times 100 \%=44 \%$ which includes Prevalence of sacralisation $=(63 / 150) \times 100 \%=42 \%$, Prevalence of lumbarization $=(3 / 150) \times 100 \%=2 \%$, Prevalence of sacralization in HNP group $=64.28 \%$, Prevalence of sacralization in non HNP group $=23.37 \%$.

Male and female ratio $=38: 25=1.52: 1$

\section{Relative Risk of Sacralization for HNP}

Odd ratio $=(\mathrm{a} / \mathrm{c})(\mathrm{b} / \mathrm{d})=\mathrm{ad} / \mathrm{bc}=45 \times 59 / 18 \times 25=5.92$. This indicates that sacralized subjects are 5.92 times risk for the HNP. Graph 2 shows distribution of different types of sacralization in HNP.

Out of total 63 subjects with sacralization (group A + group B) 45 were having HNP (group A). Table 1 shows the subjects in each types of sacralization:

Proportions of Type 2 sacralization in low back pain subjects were found to be $60.31 \%$. Type 2 sacralizations are found to be more in both LBP population and HNP population, out of them type 2a is the highest (Table 2).

\section{Pain}

One way ANOVA for pain showed statistical significant difference between group $\mathrm{A}, \mathrm{B}, \mathrm{C}, \mathrm{D}$ with $\mathrm{F}=21.558, \mathrm{P}=0.000, \mathrm{Df}=3$.

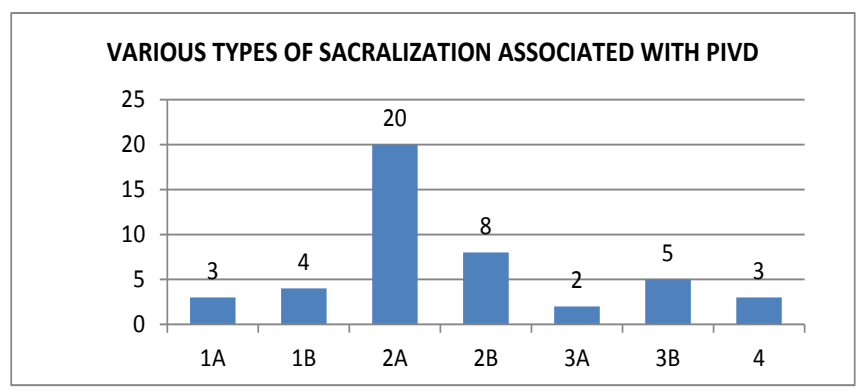

Graph 2: Distribution of different types of sacralization in HNP.

\begin{tabular}{|c|c|c|}
\hline Type of sacralization & $\begin{array}{c}\text { Number of subject with } \\
\text { sacralisation }\end{array}$ & $\begin{array}{c}\text { Number of subject with } \\
\text { HNP }\end{array}$ \\
\hline 1A & 5 & 3 \\
\hline 1B & 5 & 4 \\
\hline 2A & 25 & 20 \\
\hline 2B & 13 & 8 \\
\hline 3A & 3 & 2 \\
\hline 3B & 8 & 5 \\
\hline 4 & 4 & 3 \\
\hline
\end{tabular}

Table 1: The subjects in each types of sacralization.

\begin{tabular}{|c|c|c|c|}
\hline GROUP & $\mathbf{L}_{\mathbf{3}}-\mathbf{L}_{\mathbf{4}}$ & $\mathbf{L}_{\mathbf{4}}-\mathbf{L}_{\mathbf{5}}$ & $\mathbf{L}_{\mathbf{5}}-\mathbf{S}_{\mathbf{1}}$ \\
\hline HNP(C) & 2 & 13 & 10 \\
\hline HNP+ SACRA(A) & 2 & 32 & 11 \\
\hline 1A & & 3 & 1 \\
\hline 1B & 1 & 2 & 9 \\
\hline 2A & & 11 & \\
\hline 2B & 1 & 7 & 1 \\
\hline 3B & & 2 & \\
\hline $\mathbf{4}$ & & 4 & \\
\hline
\end{tabular}

Table 2: Sacralization and level of disc herniation.

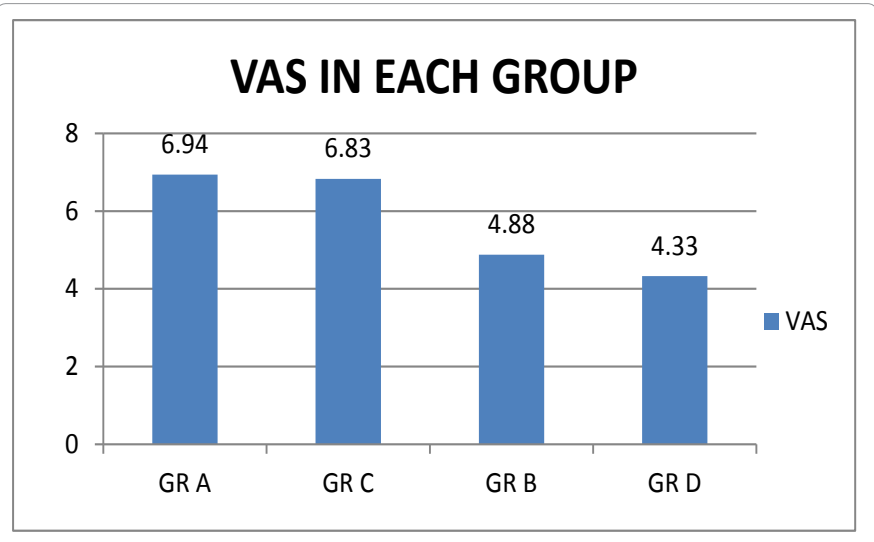

Graph 3: The mean VAS in each group.

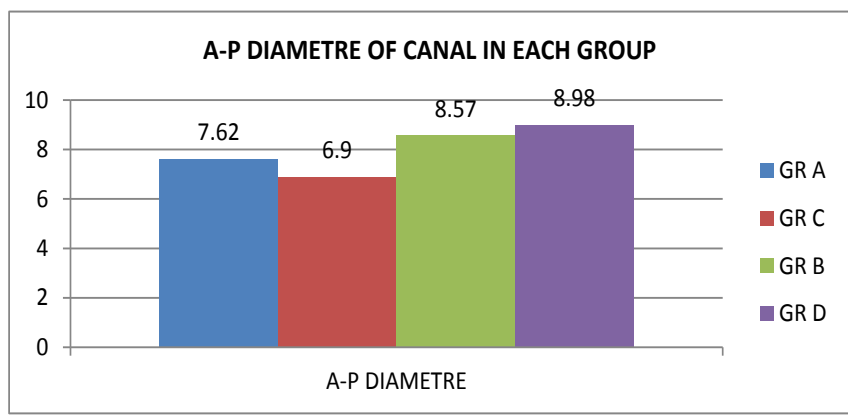

Graph 4: The mean A-P diameter of canal in each group.

Tukey's post-hoc analysis shows HNP group with and without sacralization shows mean VAS 6.94 and 6.83 respectively whereas non HNP group with and without sacralization shows 4.88 and 4.33 respectively. However, group $\mathrm{A}$ and $\mathrm{C}$, and $\mathrm{B}$ and $\mathrm{D}$ are found to be similar (Graph 3).

\section{A-P Diametre of Canal}

One way ANOVA shows no statistical significant difference in A-P diametre of canal between the groups $\mathrm{A}, \mathrm{B}, \mathrm{C}, \mathrm{D}$ with $\mathrm{F}=2.578, \mathrm{P}=$ $0.59, \mathrm{DF}=3$

Tukey's post-hoc analysis shows HNP group with and without sacralization shows mean diameter 7.62 and 6.90 respectively whereas non HNP group with and without sacralization shows 8.57 and 8.98 respectively. All the groups found to be similar in diameter (Graph 4).

\section{A-P diameter and VAS}

Pearson correlation coefficient between VAS and A-P diameter of canal is 0.123 (Graph 5).

\section{Disability}

One way ANOVA shows statistical significant difference between the groups A, B, C, D for disability by both ODI and WHODAS 2 .

- Tukey's post-hoc analysis for ODI shows the mean disability in group A is 57.64, in group B 41.77, in group C 49.36 and in group D 36.00 (Graph 6).

- Tukey's post-hoc analysis for WHODAS-2 shows mean disability in group A is 36.88 , in group B 28.00 , in Group C 33.60 and in group D 25.15. However, homogeneousity between (Group B and D) and (A and C) was found (Graph 7). 


\section{SLR and Disability}

Pearson correlation coefficient between SLR and ODI is 0.63 (Graph 8).

Pearson correlation coefficient between SLR and WHODAS-2 is 0.57 (Graph 9).

\section{Discussion}

A total number of 150 subjects with low back pain (LBP) were taken in the study as per inclusion and exclusion criteria. Out of the 150 subjects, 45 had both sacralisation and HNP, 18 had sacralizaion without HNP, 25 had HNP without sacralization, 59 had no HNP and no

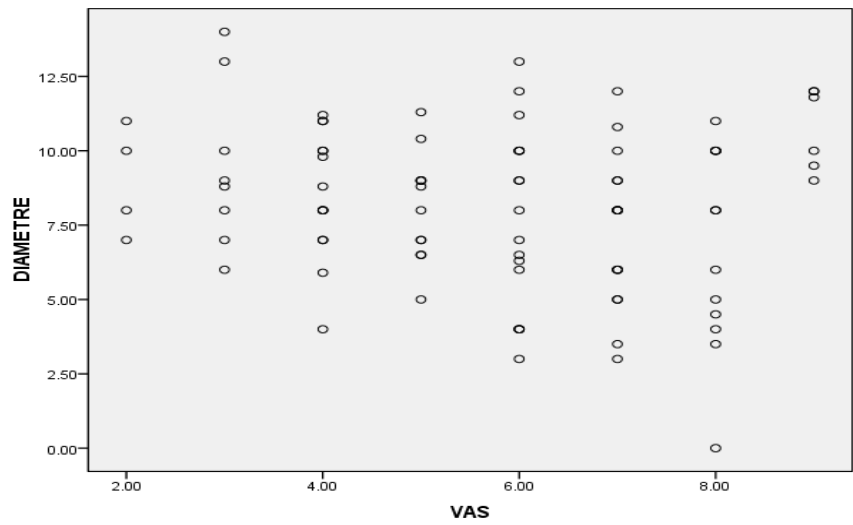

Graph 5: The correlation between A-P diameter and VAS.

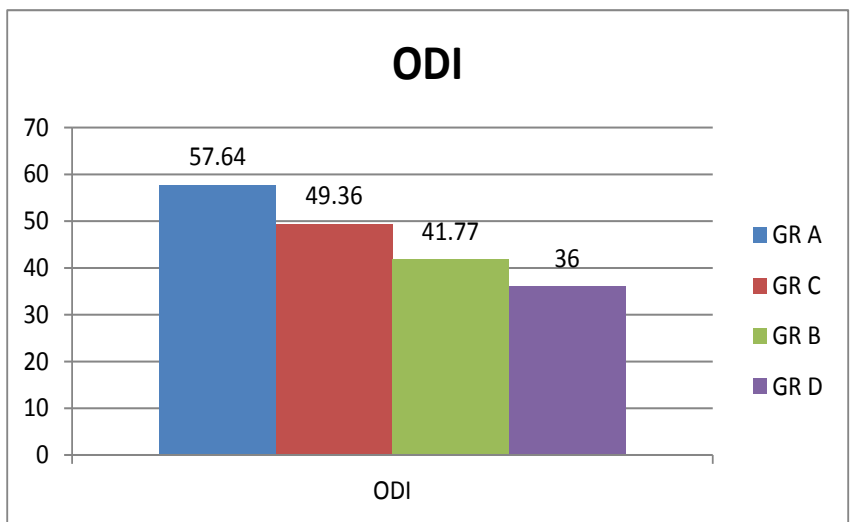

Graph 6: The mean ODI in each group.

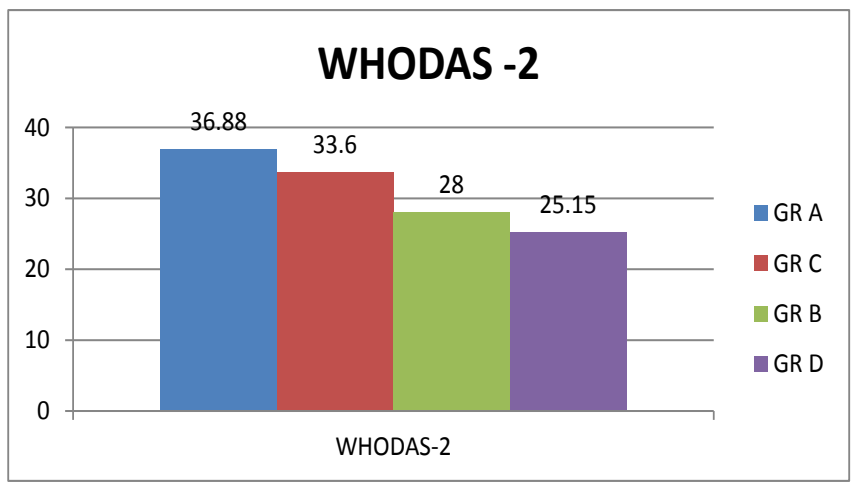

Graph 7: The mean WHODAS -2 in each group.

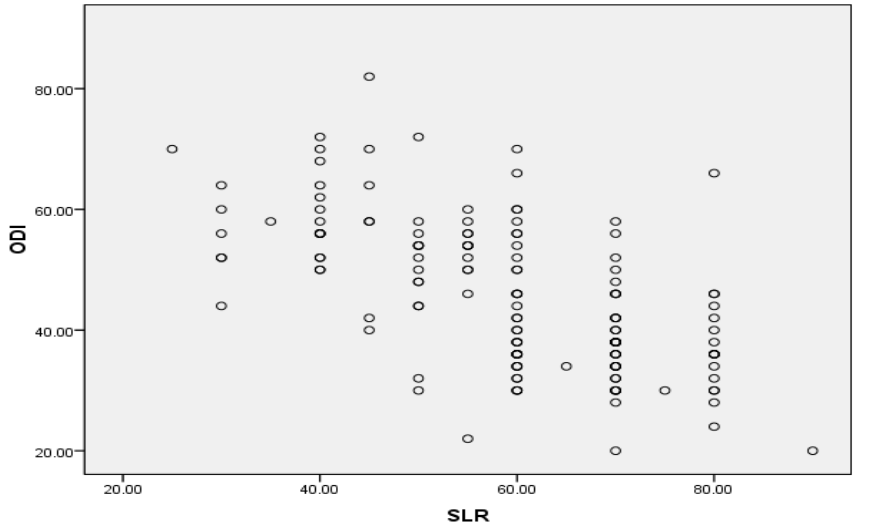

Graph 8: The correlation between SLR and ODI.

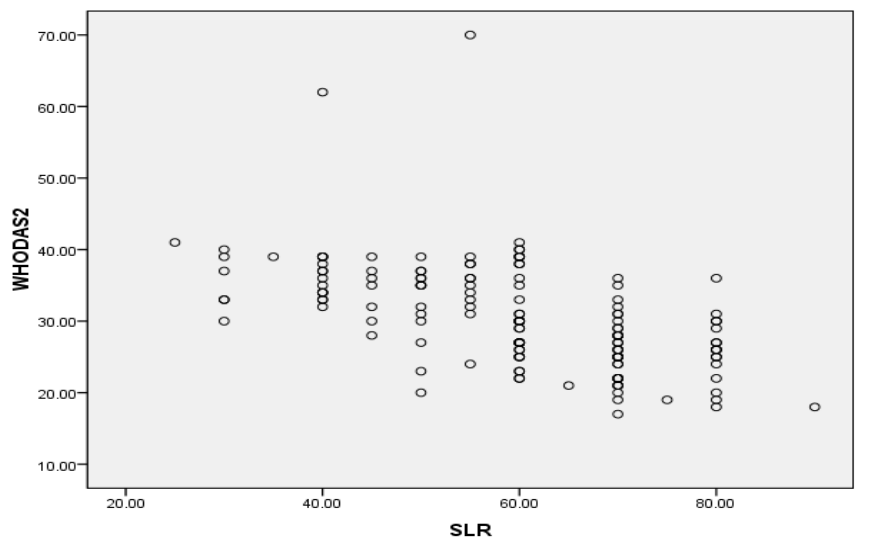

Graph 9: The correlation between SLR and WHODAS-2.

sacralisation. 3 subjects presented with LBP had lumbarisation without HNP. They were divided in to 4 groups according to the presence or absence of HNP and sacralization. Group A - HNP+ sacralisation, Group B- No HNP, sacralisation, Group C- HNP, No sacralisation and Group D - No HNP, No sacralization. The overall result of the study showed that sacralization is associated in many subjects with HNP and it is a risk factor for HNP. Besides this, it has also been found that sacralized subjects suffer from more disability and there is a weak correlation between SLR and disability. However, sacralization doesn't give rise to significant reduction of A-P diameter of spinal canal and more pain.

\section{Prevalence of sacralisation}

In this study, the prevalence of LSTV was found to be $44 \%$ which includes sacralization (42\%) and lumbarisation (2\%). Mehmut et al. [13] had found the incidence of sacralization in low back pain subjects is $21.2 \%$. Konin and Walz [14] in a review of article has reported the LSTV prevalence is $4-30 \%$ in general population. In this study, we also found the type 2 sacralizations are relatively more (60.31\%) compared to other types of sacralization. Cynthia et al. [15] in their study found 43 sacralizations out of 353 low back pain subjects, out of which 25 (58\%) were type 2 . We also found that the male: female ratio is $1.52: 1$. Singh et al. [9] in their study showed the frequency of developing sacralization is more in males $(20 \%)$ in comparision to females $(10 \%)$.

\section{Association between sacralization and HNP}

In this study, it has been found that $71.42 \%$ sacralized subjects have HNP and sacralized subjects are 5.92 times risk for HNP. 

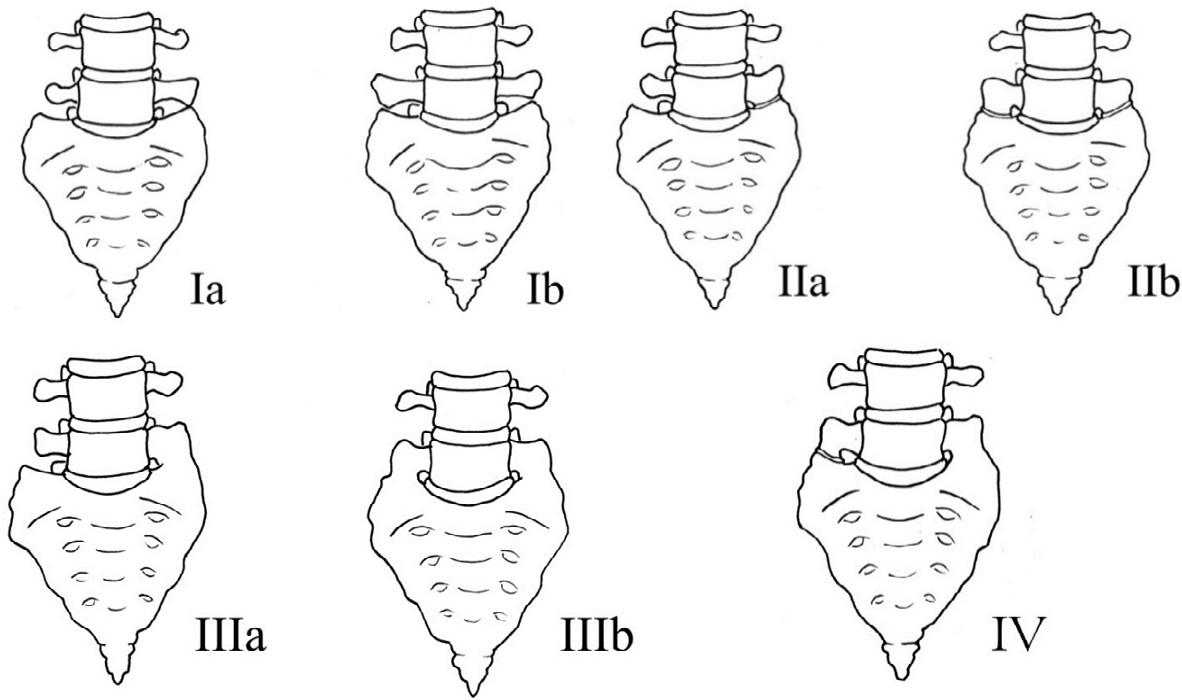

Figures 1-4: Figure shows different types of sacralization [29].

Incidence of sacralization in HNP group also found to be more i.e., 64. $28 \%$. These figures suggest that one of the risk factor for herniated nucleus pulposus is sacralisation (Figure 1-4). In this study, it has also been found that the relative risk factor for HNP in type $2 \mathrm{a}$ and $1 \mathrm{~b}$ is highest i.e., 9.44 each, next to it is type -4 i.e., 7.08.

Does sacralization is really a cause of low back pain and HNP is still a controversy in literature. Otani et al. [16] suggest that presence of TV affect the incidence of nerve root symptom caused by disc herniation. Castellvi [17] studied in 200 subjects with positive myelographic findings of herniated lumbar disc found that 60 subjects have LSTV. He had given a new classification of LSTV based on the morphological and clinical characteristics with respect to herniated nucleous pulposus. Type-1 sacralization represents 'forme furste' and shows no difference in location of herniation.

In type-3 and 4, no herniation is also seen at same level or above. Only type- 2 causes herniation at same level and above and herniation at the level just above is seen in greater extent. Similarly in this study we had found that type 2a sacralization causes herniation at L5-S1 as well as a level above L4-L5, whereas in other types we found L4-L5 herniation is more common. In type 2a sacralization, the L5-S1 disc herniation can be explained by the following reasons. Asymmetrical intervertebral joint articulation and movement in lumbar spine put stress on disc, forcing fluid nucleus laterally lead to scoliosis [5]. Asymmetrical movement results in pressure exerted by nucleus in a part of annulus. This portion of annulus degenerate and tear prematurely lead to disc herniation. Kessler and Hertling [11] suggested that unilateral contact put unusual stress on spine and results in torque movements cause herniation of disc one level above the sacralization. Another thing to consider is that the discs of the spine act as shock absorbers; having one less disc may result in extra jarring of the spine.

If other discs must take on more pressure due to the lack of a L5 disc, then it is possible that they will wear faster [18]. Elster [19] had also mentioned hypermobility of disc above and below occur following post-surgical spinal fusion and block vertebra same as LSTV. Various authors have advocated that hypermobility and abnormal torque movements at intervertebral disc place the disc and facet increased risk of accelerated degeneration [14]. These are the reasons why L4-L5 disc herniation also found in type-2A sacralization subjects.
Furthermore, when we have compared the HNP and HNP + sacralization group we found that L4-L5 disc herniation is more common in HNP + Sacralization group. Many authors have reported increased disc degeneration and herniation above the TV. Aihara et al. [12] found that the illiolumbar ligament at the level immediately above is thinner and weaker whereas at the level of LSTV is broader and stronger. Because of this weak illiolumbar ligament the disc above the level of LSTV undergoes degeneration. Below to it stability is preserved due to bony union and protect disc from injury. He had studied 52 subjects and found above the transitional vertebra significantly more degenerative.

\section{Relation between sacralization and pain}

The mean pain in sacralized subjects is 5.91 and in non sacralized subjects are 5.58. Cynthia et al. [20] in a cross sectional study had found no significant difference in pain and disability level with or without transitional vertebra. In subjects with HNP with and without sacralization the mean difference in VAS is 0.10 whereas in non HNP groups with and without sacralization is 0.55 . These findings suggest that non HNP subjects with sacralization suffer from little more pain compared to non-sacralised subjects. Peterson et al. [21] had found lumbar spine degeneration has a weak correlation with pain. Many studies had investigated the link between degeneration and pain and found conflicting result. But the weights of these evidences suggest that subjects with low back pain and degeneration have slight increase in pain in standing. Sacralization alters the spinal biomechanics. It gives rise to early degenerative disc disorder, facet joint arthritis, ligamentous strain [22]. This might be another reason why sacralized individual had slightly more pain.

\section{Relation between sacralization and disability}

The mean disability in group A is 57.64 , in group B 41.77 , in group C 49.36 and in group D 36.00. ODI indicates that HNP with sacralized subjects suffers from greater disability compared to HNP without sacralization, whereas non HNP subjects with sacralization have greater disability compared to non sacralized subjects. In low back pain population, the mean disability in sacralized individuals found to be 49.70 whereas in non sacralized individual is 42.68 ; suggest that sacralization gives rise to more disability. HNP itself give rise to a 
greater disability. Porchet et al. [23] had found a positive correlation of disc disorder with disability. The separation of vertebrae in the spine allows for mobility and flexibility. It stands to reason that those with one less movable vertebra will exhibit a slight reduction in range of motion. [18] Out of $219^{\circ}$ flexion and extension approximately $70-75 \%$ of lumbar flexion occurs at lumbosacral junction, $20-25 \%$ at L4-L5 and 5-10\% at L1-L3. Limited mobility following sacralization can be one of the reasons why sacralized subjects have greater disability. In ODI most of the items like sitting, standing, walking, travelling, lifting were physical in nature. HNP subjects experience more pain as shown by VAS so also they exhibit greater limitation of ROM of lumbar spine. When sacralization is added with HNP they exhibit further more limitation of ROM which is reflected in their physical performance shown by ODI.

Tukey's post-hoc analysis for WHODAS-2 shows homogeneousity between Groups B and D and A and C is found. The mean difference in the HNP group between sacralized and non- sacralized subjects is 3.28 and non HNP subjects with and without sacralization is 2.84 only. In low back pain population, the mean disability in sacralized individuals found to be 32.44 whereas in non sacralized individual is 29.37 suggest no significant difference. WHODAS 2 is a 12 item score which contain physical, social, emotional, cognitive aspects. Though sacralization has an effect on physical performance but it mayn't have a social and emotional effect. Therefore no significant difference in disability between sacralized and non sacralized subjects has found by WHODAS 2.

\section{Relation between SLR and disability}

In this study, SLR and disability was weakly correlated. Pearson correlation coefficient between SLR and ODI is 0.63 and between SLR and WHODAS-2 is 0.57 . Straight leg raising is frequently used as in the assessment of patients presented with lumbar spine dysfunction and pain. Sharma and Bhavsar [24] in their study found that angle of SLR where tingling and pain starts plays an important role to know the disability. It has also been suggested that improving the range of hip flexion has a beneficial effect in restoring normal movement and reducing the degree of impairment due to low back dysfunction [25]. Pawar and Metgod [26] in a randomized control trial used manual therapy technique to increase the SLR and found improvement of pain, ROM and reduction of disability in low back pain subjects. In this present study we found HNP subjects had a limited SLR and presented with grater disability compared to non HNP subjects. This also suggests the correlation between disability and SLR. Limited SLR affects the physical performance which is reflected in ODI and WHODAS-2.

\section{Relationship between sacralization and A-P diameter canal}

Tukey's post-hoc analysis showed mean A-P diameter in sacralized individual found to be 8.09 and in non sacralized individual found to be 7.94 showed that no significant difference in diameter. Vergauwen et al. [27] didn't found no statistical significant difference in spinal canal stenosis between with and without LSTV subjects. Oguz et al. [28] had also found no relation between the spinal canal diameter at adjacent level and LSTV.

\section{Relation between A-P diameter and pain}

Pearson correlation coefficient between VAS and A-P diameter of canal is found to be 0.123 . When A-P diameter is correlated with VAS no statistical significant correlation was found. In the present study, it has been found that HNP with sacralization subjects demonstrate greater pain and disability compared to HNP alone. But mean diameter of HNP without sacralized subjects is slightly more than HNP with sacralized subjects. It has been said that though degeneration is the cause of low back pain but no evidence is found radiologically, and many who have degeneration radiologically do not demonstrate back pain [23]. This also found to be true here for the relationship between A-P diameter of canal and back pain.

\section{Conclusion}

The study found that sacralization is associated with Herniated nucleus pulposus and L4-L5 disc prolapsed was common in sacralised subjects. Sacralization is a risk factor for HNP and out of all sacralization type $-1 \mathrm{~B}$ and $2 \mathrm{~A}$ risk factor is highest. It has also been found that prevalence of sacralization in the low back pain population is more, sacralized individual suffered from more disability but not more pain or any changes in A-P diameter of spinal canal. SLR is weakly correlated with disability, A-P diameter of spinal canal and pain are not correlated.

\section{Limitations}

Small sample size

\section{Future Recommendations}

- Sacralized individual without HNP can be follow up to know whether they suffering from HNP in later part of life.

- Experimental study can be done in HNP with and without sacralized subjects.

\section{References}

1. Hoy D, Brook P, Blyth F, Buchbinder R (2010) The epidemiology of low back pain. Best Pract Res Clin Rheumatol 24: 769-781.

2. Chou R (2010) Low back pain (chronic). BMJ Clin Evid 1116.

3. Calliet $R$ (1981) Low back pain syndrome. Pain series

4. Lunawat SK, Taneja DK, Malviya A (2002) Correlation between MRI and intraoperative finding in prolapsed intervertebral disc. Indian J Orthop 36: 12.

5. Mckenzie RA (1981) The lumbar spine mechanical diagnosis and therapy. Spinal Publications New Zealand Limited, Waikanae, Wellington, New Zealand.

6. Cyriax J (1982) Text book of orthopedic medicine (8thedn) Volume onediagnosis of soft tissue lesion. Bailliere Tindall.

7. Khairnar K, Rajale MB (2013) Sacralization of lumbar vertebra. Int J App Basic Med Res 2: 510-514.

8. Sharma VA, Sharma DK, Shukla CK (2011) Osteogenic study of lumbosacra transitional vertebra in central India region. J Anat Soc India 60: 212-217.

9. Singh R (2014) Classification and analysis of fifth pair of sacral foramina in indian dry sacra. Int j morphol 32: 125-130.

10. Cox JM (2011) Low back pain; mechanism, diagnosis and treatment (7thedn). Lippincott Williams \& Wilkins.

11. Hertling D, Randolph M (1996) Management of common musculoskeletal disorder (3rdedn). Lipincott William \& Wilkin.

12. Aihara T, Takahashi $\mathrm{K}$, Ogasawara A, Itadera $\mathrm{E}$, Ono $\mathrm{Y}$, et al. (2005) Intervertebral disc degeneration associated with lumbosacral transitional vertebrae: a clinical and anatomical study. J Bone Joint Surg 87-B: 687-691.

13. Bulut M, Ucar BY, Ucar D, Azboy I, Demirtas A, et al. (2013) Is sacralization is really a cause of Low back pain. ISRN orthopedics 839013

14. Konin GP, Walz DM (2010) Lumbosacral transitional vertebrae: Classification, imaging findings, and clinical relevance. AJNR Am J Neuroradiol 31: 1778-86.

15. Peterson CK, Bolton J, Hsu W (2004) A cross-sectional study comparing pain and disability levels in patients with low back pain with or without transitional vertebra. J Manipulative Physiol Ther 28: 570-574.

16. Otani K, Konno S, Kikuchi S (2001) Lumbosacral transitional vertebrae and nerve-root symptoms. J Bone Joint 83B: 1137-1140.

17. Castellvi AE, Goldstein LA, Chan DPK (1984) Lumbosacral transitiona vertebrae and their relationship with lumbar extradural defects. Spine 9: 493495 . 
18. Back posture correction via Wristband. Back pain solutions online. Accessed on: 13 April 2016.

19. Elster AD (1989) Bertolotti's syndrome revisited: transitional vertebrae of the lumbar spine. Spine 14: 1373-1377.

20. Peterson CK, Bolten JE, Wood AR (2000) A cross sectional study correlating lumbar spine degeneration with pain and disability. Spine 25: 218-223.

21. Singh AP, Sekhon J, Kaur N (2014) Sacralization-the structural complications and body biomechanics. Human Biology Review 3: 88-94.

22. Wani SK, Deshpande N (2014) Correlation of pain and disability with mri findings in patients with lumbar discogenic back pain. Int $\mathrm{J}$ Physiother Res 2 : 418-423.

23. Sharma AA, Bhavsar $U$ (2012) Straight leg angle and low back pain paperback. LAP Lambert Academic Publishing.
24. Hall T, Beyerlein C, Hansson U, Lim HT, Odermark M, et al. (2006) Mulligan traction straight leg raise: A pilot study to investigate the effects on range of motion in patients with low back pain. J Man Manip Ther 14: 95-100.

25. Pawar AH, Metgud S (2012) Comparative effectiveness of Mulligan's traction straight leg raise and bent leg raise in low back ache with radiculopathy - A randomized clinical trial. Int J Health Sci Res 3: 6-10

26. Vergauwen S, Parizel PM, van Breusegem L, Van Goethem JW, Nackaerts $Y$, et al. (1997) Distribution and incidence of degenerative spine changes in patients with a lumbo-sacral transitional vertebra. Eur Spine J 6: 168-172.

27. Oguz H, Akkus S, Tarhan S, Açikgözog lu S, Kerman M (2002) Measurement of spinal canal diameters in young subjects with lumbosacral transitional vertebra. Eur Spine J 11: 115-118.

28. Konin GP, Walz DM (2010) Lumbosacral transitional vertebrae: classification imaging findings, and clinical relevance. AJNR Am J Neuroradiol 31: 1778-1786. 